\section{Method of constant stimuli: Recalculations show that response-frequency equalization cannot fit the data*}

\author{
FRANK RESTLE† \\ Indiana University, Bloomington, Indiana 47401
}

The bias found in the method of constant stimuli has been attributed either to an adaptation-level effect or to a response bias. Restle and Levison presented data purported to show that the result could not be explained by response frequency equalization, the best available response-bias theory. Erlebacher and Sekuler elaborated their theory and calculated a version of the model that would fit the main experimental result, but chose an erroneously large value for the standard deviation of their model. Their model does not produce an acceptable fit to the data, and therefore does not successfully compete with the adaptation-level model.

In a recent exchange of studies, agreeing that the method of constant stimuli is dangerously biased but disagreeing as to the mechanism, Restle and Levison (1971) reported an experiment purported to disprove the model of response frequency equalization (RFE) as an explanation for this bias. Erlebacher and Sekuler (1971) responded with a paper formulating their RFE theory into a mathematical model, and from it computed predictions of the Restle-Levison data. The purpose of this paper is to point out that an unfortunate choice of estimators, in the calculations reported by Erlebacher and Sekuler, led to an erroneous conclusion.

The RFE theory derives from classical psychophysics. When the $O$ compares a given comparison stimulus (Co) with the standard (Std), the resulting comparison may be in any of three categories: Co may be perceptibly smaller, it may be perceptibly larger, or the difference may be uncertain. In the first two cases, the $O$ makes the appropriate response, but when the result is uncertain, in some band about the point of subjective equality (PSE), the O may say either "smaller" or "greater." In this uncertain region, the $O$ adjusts his response bias or probability of saying "greater," so as to equalize the overall frequency of responses "smaller" and "greater" in the session.

To test the RFE theory, it is only necessary to locate the standard deviation of the true underlying

*This study was supported in part by Resenrch Grant MH 16817 from the U.S. Research Grant MH

tRequests for reprints should be sent to the author at the Department of Psychology, Indiana University. Bloomington, Indiana 47401. psychometric functions and the two thresholds, $\mathbf{T}_{\mathbf{L}}$ and $\mathrm{T}_{\mathbf{U}}$, that separate the interval of uncertainty from the lower and upper regions, respectively. This is not a simple problem, however, since none of these parameters is reflected directly in the observed psychometric function.

The first point to establish is that Erlebacher and Sekuler did not, in fact, fit the Restle-Levison data. This is shown by comparing their computed predictions (from their Table 2, p. 317) with the observed psychometric functions given in their Table 1 (p. 316). They were able to find parameters for which the apparent PSEs of the two groups differ by the correct amount and in the correct direction-but their predictions are strikingly far off at every other point, showing that they have chosen much too large a value of SD (see Fig. 1).

We have attempted to improve upon the estimates of Erlebacher and Sekuler in several ways. Mainly, we ave used STEPIT, 1 a funct on-maximizing computer routine, to compute minimum chi-squared estimates of the parameters of the two psychometric functions. We find that $S D$ is estimated at approximately 1.25 and 1.30 , respectively, for the two groups. These values are far lower than estimated by Erlebacher and Sekuler, who obtained 2.46 and 1.96 from two parts of the data and adopted a value of 2.21. When they searched the parameter space to evaluate their model (Erlebacher \& Sekuler, 1971 . Figs. 3, 4, and 5), they varied SD only from 1.75 to 2.75 and found that their model made wrong predictions at $S D=$ 1.75. We believe that with $\mathrm{SD}=1.25$ or 1.3 , their model will consistently fail to predict the observed results of our experiment.

\section{CONCLUSION}

Erlebacher and Sekuler (1971) made two errors in their calculations. First, they estimated parameters from extreme values of a psychometric function and by methods with somewhat uncertain logic. The result was quite poor estimates of crucial parameters. Second, they failed to test whether their model correctly reproduced the overall properties of the data (in particular, the shape and

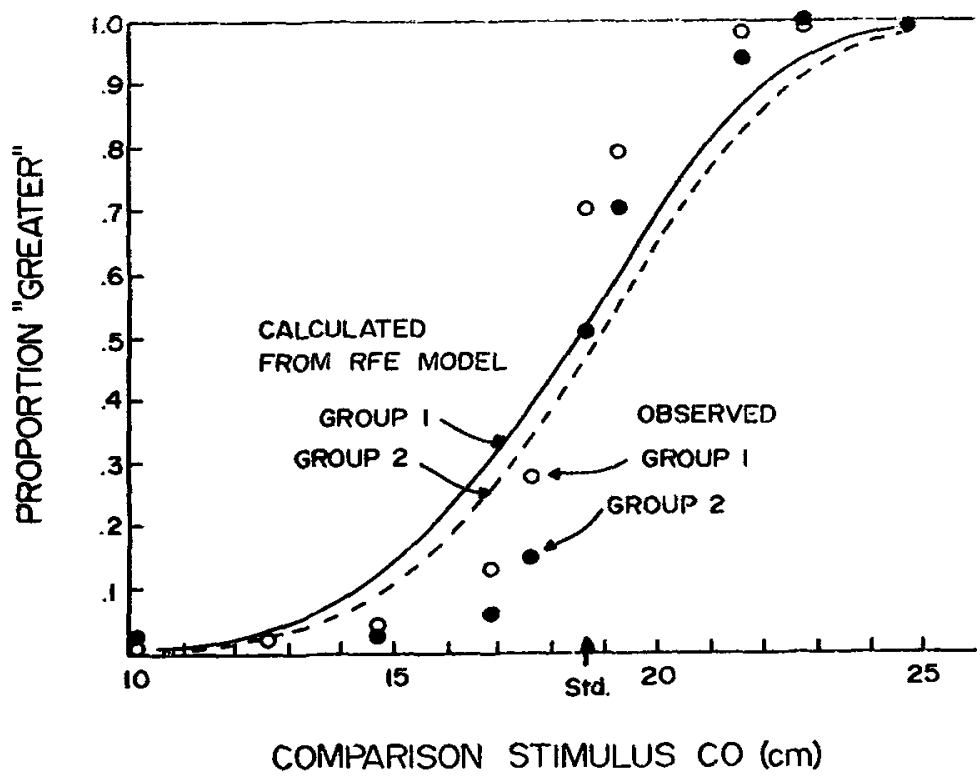

Fig. 1. 
position of the psychometric functions), even though the necessary data were all in their paper. As a result, they overlooked the discrepancy shown in our Fig. 1.

With these very wrong estimates, Erlebacher and Sekuler were then able to fit their RFE model to the Restle-Levison data-more exactly, they were able to find a case of the RFE model's having the same discrepancy at PSE between Groups 1 and 2 as in our data. Since their model definitely does not fit the data as a whole, their fitting of particular points is not convincing. It is my belief at this time that the RFE model as formulated by Erlebacher and Sekuler cannot be reconciled with the results of the Restle-Levison experiment.

It is perhaps unnecessary to reiterate that the results were correctly predicted by adaptation-level theory, based upon comparison adaptation level. As it stands, the experimental result does distinguish between the two models; it agrees with the adaptation-level model and disagrees with the model of response frequency equalization.

REFERENCES

ERLEBACHER, A., \& SEKULER, R. (Received for publication June 5, 1972.)
Response frequency equalization: A bias model for psychophysics. Perception \& Psychophysics, 1971, 9, 315-320.

RESTLE, F.. \& LEVISON, M. Method of constant stimuli: Invalidity to the third power. Perception \& Psychophysics, $1971,9,312-314$.

\section{NOTE}

1. Subroutine STEPIT, copyrighted in 1965 by J. P. Chandler, Physics Department, Indiana University. The author would like to thank Steven P. Edgell for performing a variety of calculations in our attempts to fit the RFE model to the Restle-Levison data. 